\title{
Industrial/Organization Consultancy in the United States: A Few of the Challenges
}

\author{
Barbara Nett ${ }^{1}$ \\ Angie Rosenbaum
}

As Industrial/Organizational (I/O) consultants, we are normally hired by a human resources department (e.g., staffing, talent management) within a large organization or the legal department. We are typically brought in to deal with an organizational issue that the internal staff cannot handle because they lack the necessary staff, time, and/or technical expertise. That means we are viewed as people who have all the answers needed to get the job done, and can get it done quickly. By the time we are brought on board, the clients are eager to get the project underway and are thrilled to be able to ask their questions and listen to our advice. However, as the projects progress, any number of challenges may arise.

Credibility. It is not unusual for the client's project staff to be curious about our credibility. Our Chief Executive Officer speaks to their figure head to secure the contract, and then we come in to assist with the work. We may have met the executive staff and gained their acceptance, but when we first meet the project staff, they are inquisitive. They quiz us with questions such as, "How long have you been doing this type of work? How many similar projects have you worked on? What other clients have you worked for?" and so on. Once we explain we have worked in the field for a dozen or more years and have an impressive list of clients, they feel they have sufficiently vetted us and they become more at ease partnering with us. We imagine this establishes for them that we not only have the necessary technical expertise, but that we have also actually used it successfully - and we may actually be able to help them!

Eventually, a funny thing happens. Once we have earned their trust and confidence, the clients become so comfortable relying on us to provide advice regarding their dilemmas and answers to their questions that they become reluctant to make routine decisions for themselves - even the decisions they were making regularly before they engaged us. For instance, after developing a

\footnotetext{
${ }^{1}$ Adresa de corespondență: blnett@comcast.net
}

new selection process for a client, they asked us "Where should we post our openings?" Noting that recruitment had never been an issue for them, we responded with "Where have you posted them in the past?"

As another example, we often recommend that clients use banding rather than selection based on strict rank order. After developing a new selection system for a large public safety organization we recommended they band the test scores. The client asked us to then suggest how they should decide which candidates to select from within the bands. We advised that they could make selections from within the bands using any number of jobrelated criteria such as seniority, top-down based on interview score, geographic preference, etc. - as long as the criteria were consistently applied. Yet, the client kept pressing us for a firm recommendation - and we kept insisting that it was ultimately their decision to make based on what fit the needs of their business.

Setting Limits. Our impression is that organizations are slow to pull the trigger on hiring external consultants. And once they do they want the work completed at lightning speed. The organizational issue at hand has probably been lingering for some time and now needs immediate attention. So in their haste to accomplish the project clients try to push the limits - sometimes wanting to sacrifice sound scientific methodology in the name of cost containment and quick results. We try to be amenable to making logistic concessions and rearranging our schedules, but never bend to the pressure if it means sacrificing unassailable methodological practices.

Additionally, client questions frequently segue into requests beyond the scope of the initial work, with no mention of additional compensation or flexibility of deadline. Clients are tempted to sweep peripheral organizational issues under the rug of our contract agreement. We have to be willing to say "no" sometimes and remain vigilant not to be taken advantage of while not compromising our relationship with the client. 
Packaging Technical Expertise. Hired for our expertise, challenges arise in conveying that expertise to the client in an understandable fashion. Issues such as banding, setting effective and defensible cutoff scores, why to use compensatory vs. multiplehurdle testing systems, and even what constitutes a "test" can be surprisingly difficult for clients to comprehend. One client, when asked what type of test they used for selecting clerical staff, said "We don't use a test instead, we just have them come in to provide a typing sample." Many clients do not understand that any mechanism used in a pass/fail or recommend/not recommend fashion is considered a test. It's also not uncommon to encounter clients who still do not understand that an interview is a test.

We also encounter clients who do not understand that selection is an imperfect science and that testing for each and every competency may be both cost-prohibitive and inefficient. They have trouble recognizing that a parsimonious selection system is preferable, and beyond incorporating those competencies with the most predictive value, there is likely a point of diminishing returns. Similarly, clients may ask us to include selection measures for competencies that either do not survive the job analysis or that may be related to broad business initiatives but aren't necessarily jobrelated for the job in question. Just this week a client asked us to include measures for initiative and leadership in a selection procedure for an entry-level manufacturing position - two competencies not supported by the job analysis undertaken for the position.

Organizational Dynamics. Recently, we were hired by a large retailer to undertake a massive job analysis and selection project. We negotiated and agreed to an aggressive timeline for the ambitious project. Then, following disappointing quarterly results for the retailer, they received pushback from the executives to minimize costs. Their first reaction was to try to persuade us to limit the scope of the project - implying we could cut corners (by considerably reducing the job analysis sample) and still achieve the same results. We disagreed, citing the importance of a solid job analysis for any subsequent development of selection instruments. We then renegotiated the project and agreed to a modified fixed-price contract for the work. Again, just before the project was set to launch, they halted our efforts in favor of having a sister group within the organization help with the travel-intensive project in order to further control costs.
Sometimes this on-again off-again work is related to individuals, not resources. Occasionally, an executive who brings us on for a project exits the organization and support for the project disappears as well. We have learned to enjoy down-time and lenient schedules when we can, because we can never count on how long it will last and need to be prepared to change gears on a moment's notice.

Schedules at the Mercy of the Client. Given how quickly our projects may come and go, we are required to be exceedingly flexible in terms of schedule. A project may arise quickly and have a very tight deadline. In those instances, it may be necessary to work weekends and holidays to accomplish the project. Many of our projects are also extremely labor-intensive and require long and arduous hours at the client site. We have spent as many as six consecutive weeks at a client site (in another state) in order to meet project deadlines. And on one particular job analysis project, we worked 20-hour shifts in order to adequately sample all shifts in the $24 / 7$ production facility. The unpredictability of our work and the frequent work/life imbalance some of the greatest obstacles we encounter in retaining staff. Consulting requires tremendous dedication and commitment to the work and many don't want the occasionally demanding lifestyle.

Long-Term Client Relationships. Ideally, we aim to secure the trust and confidence of our clients so that we can establish a long-term relationship and gain repeat business with them. It is generally advantageous for all parties involved. Familiarity generally breeds efficiency in that the parties involved already know one another, we understand the organizational culture and protocol, and we may be able to build on earlier project work. One of the most professionally gratifying feelings is to gain repeated business with a client - that's when we know we have delivered solid work and managed to successfully overcome any challenges along the way. 